Evolution of Massive Stars, Mass Loss and Winds

M. Heydari-Malayeri, Ph. Stee and J.-P. Zahn

EAS Publications Series, Vol. 13 (2004) 293-316

\title{
MASSIVE BINARIES AND COLLIDING WINDS
}

\author{
G. Rauw ${ }^{1}$
}

\begin{abstract}
In this contribution, I review recent results from observational investigations of early-type binaries of spectral types $\mathrm{O}$ and WolfRayet. These studies aim at an accurate determination of fundamental parameters such as masses and radii of the most massive stars. In addition, multi-wavelength analyses of wind collisions in massive binaries provide constraints on the properties of stellar winds of massive stars and shock physics of cosmic plasmas.
\end{abstract}

\section{Introduction}

Although they are rather rare objects, massive early-type stars (initial masses exceeding $20 \mathrm{M}_{\odot}$ ) of spectral types $\mathrm{O}$ and Wolf-Rayet (WR) play a key role in the evolution of the galaxies. In fact, they are the hottest and most luminous objects of young stellar populations and account therefore for the bulk of the light emitted by distant starburst galaxies. Their stellar winds carry huge quantities of kinetic energy thereby shaping the ambient interstellar medium. Evolved early-type stars release huge amounts of chemically enriched material into space.

Despite considerable progress in our understanding of the physics of these stars, many questions, related to the evolution of these objects and the properties of their winds, still remain unanswered. A number of these questions can be addressed through the study of massive binaries.

This review is organised as follows. Section 2 summarizes some general results obtained from the study of $\mathrm{O}+\mathrm{O}$ and $\mathrm{WR}+\mathrm{O}$ systems. Sections 2.1 to 2.4 illustrate a few aspects of the study of massive binaries: how do the mass determinations of stars in binaries compare to other (indirect) techniques, what can we learn about the properties of open clusters harbouring massive binaries, what are the most massive stars in binaries and how can we identify systems that have experienced mass exchange? Section 3 presents a brief introduction to the concept of interacting winds in early-type binaries as well as some observational evidence ${ }^{1}$ Institut d'Astrophysique et de Géophysique, Université de Liège, Allée du 6 Août 17, Bât
B5c, 4000 Liège (Sart-Tilman), Belgium

(C) EAS, EDP Sciences 2003 DOI: $10.1051 /$ eas:2004058 
for this phenomenon at various wavelengths (from the radio to the X-ray domain). Finally, some conclusions are given in the last section.

\section{Early-type spectroscopic binaries}

The study of binary systems provides the only direct means to measure the masses of stars all across the Hertzsprung-Russell diagram. If the spectrum of a binary system displays lines belonging to only one of the components, we are dealing with a single-lined spectroscopic binary (SB1). For this category of binaries, measuring the Doppler shift of the lines as a function of the orbital phase allows us to establish the radial velocity curve of the brightest binary component (generally the primary ${ }^{1}$ ) only. The analysis of this radial velocity curve yields the mass function:

$$
f(m)=\frac{m_{2}^{3} \sin ^{3} i}{\left(m_{1}+m_{2}\right)^{2}}=1.036 \times 10^{-7} K^{3} P_{\text {orb }}\left(1-e^{2}\right)^{3 / 2}
$$

where $m_{1}$ and $m_{2}$ are the masses (in $\mathrm{M}_{\odot}$ ) respectively of the primary and secondary component, $i$ is the inclination of the orbital plane with respect to the plane of the sky. $K, P_{\text {orb }}$ and $e$ indicate respectively the amplitude of the radial velocity curve (in $\mathrm{km} \mathrm{s}^{-1}$ ), the orbital period (in days) and the eccentricity. The mass function contains the only information on the masses of the stars in an SB1 system that can be extracted from the analysis of its radial velocities.

If the spectral signatures of both binary components are visible in the spectrum, we are dealing with an SB2 system. In this case, the simultaneous analysis of the radial velocity curves of both stars allows to derive the values of $m_{1} \sin ^{3} i$ and $m_{2} \sin ^{3} i$, i.e. the absolute masses of the stars multiplied by a factor $\sin ^{3} i$. The main unknown in this case becomes therefore the orbital inclination $i$. This parameter can be determined from an analysis of the light curve of the binary provided that $i$ is sufficiently close to $90^{\circ}$ so that the binary displays photometric eclipses. In some non-eclipsing close binary systems, it is still possible to constrain $i$ by analysing the light variations due to the changing orientation of tidally deformed stars in so-called ellipsoidal variable stars.

Therefore, eclipsing SB2 binaries provide the main source of direct measurements of stellar masses and radii that serve as test benches for the predictions of the stellar evolution theory. Among the most outstanding results of the investigation of binary systems is the discovery of an empirical mass-luminosity relation for main-sequence stars across the Hertzsprung-Russell diagram. However, for stars more massive than $\sim 25 \mathrm{M}_{\odot}$, mass loss through stellar winds considerably affects the stellar evolution even during the main sequence lifetime (see e.g. Maeder \& Meynet 1994). The relationship between the mass and the luminosity of a single massive star will therefore depend on its evolutionary status (not to speak

\footnotetext{
${ }^{1}$ Throughout this review, we shall refer to the primary as the more massive component of the binary as observed in its present-day configuration. Conversely, the secondary is the binary component which has currently the lower mass.
} 
about the effects of mass exchange in binary systems that are addressed by D. Vanbeveren in his review).

In order to provide useful constraints on the fundamental properties of single massive stars, an early-type binary system has to meet several criteria: i) it must be of type SB2, ii) it should display eclipses, iii) the stars should be inside their Roche lobes and iv) the components must be young objects that have not yet suffered any mass exchange through Roche lobe overflow. However, due to the short duration of the main-sequence phase of a massive star (a few million years), the number of observable well detached binaries among the massive systems with short orbital periods is considerably reduced. Conversely, many short period O + $\mathrm{O}$ binaries are observed in a stage of their evolution affected by a previous episode of mass exchanges (e.g. Penny et al. 1996, Rauw et al. 2002b). Mass transfer in a massive binary can be considered roughly instantaneous since it occurs on a time scale of only about $\sim 10000$ years. However, the rate of mass transfer during this short-lived interaction phase is of order $10^{-3}$ to $10^{-2} \mathrm{M}_{\odot} \mathrm{yr}^{-1}$ and the result is that the analysis of these binaries after mass exchange will no longer provide information on the properties of single massive stars.

The number of suitable $\mathrm{O}+\mathrm{O}$ systems is therefore rather low and until the early 1990's, accurate orbital elements for many of them were lacking. The main problems that prevented high quality orbital solutions from being established for a long time were the line-blending mainly due to the rotational broadening of the spectral lines and the contamination of absorption lines by emission lines formed in the stellar wind. Considerable progress has been achieved with the advent of cross-correlation techniques and the possibility of simultaneous multiple Gaussian fitting. In $\mathrm{WR}+\mathrm{O}$ binaries an additional difficulty arises from the fact that the spectra of WR stars contain no photospheric absorption lines and one has therefore to rely on the radial velocities of broad emission lines to describe their orbital motion. It has to be noted that the orbital parameters of WR stars are sometimes extremely sensitive to the choice of the spectral line adopted for the orbital solution. These effects reflect the relative motion of the line forming regions that are located in the outflowing stellar wind rather than at the level of the WR core.

Generally speaking, the study of massive binaries requires a considerable amount of observations, especially when the orbital periods are very long or ill-conditioned (i.e. shorter than about five days and very close to an integer number of days). Several teams have contributed to the recent effort aiming at a better understanding of the properties of massive binaries. A (by no means exhaustive) list of these teams includes the groups from La Plata (Argentina, e.g. Morrell et al. 2001), Atlanta (USA, e.g. Bagnuolo et al. 1994), St. Andrews (United Kingdom, e.g. Harries et al. 1998), Chilton (United Kingdom, e.g. Stickland et al. 1995), Montréal (Canada, e.g. Lamontagne et al. 1996) and Liège (Belgium, e.g. Rauw et al. 2001a).

Recent compilations of galactic $\mathrm{O}+\mathrm{OB}$ and $\mathrm{WR}+\mathrm{OB}$ binaries for which relatively accurate determinations of the masses exist can be found in Gies (2003) and van der Hucht (2001). 


\subsection{The mass discrepancy}

In the absence of an empirical mass-luminosity relation, there are two independent indirect techniques to estimate the mass of a single early-type star. The first approach consists in comparing the position of the star (i.e. its luminosity and effective temperature) in an empirical Hertzsprung-Russell diagram with the predictions of stellar evolution calculations. The second method is based on the modeling of the observed spectrum with a stellar atmosphere code. This modeling yields several stellar parameters including the radius and the gravity and hence the stellar mass (see also the review by P. Crowther in this volume).

However, the model atmosphere codes yield masses that are systematically lower than those inferred from the comparison with the evolutionary models (see e.g. Herrero et al. 1992, 2000). This discrepancy mostly affects supergiants that are close to the Eddington limit, but it also exists for O-stars on the main sequence. Herrero et al. (2000) pointed out that the use of spherical model atmospheres accounting for mass loss reduces the mass discrepancy but the spectroscopic masses are still systematically lower than the evolutionary ones.

Burkholder et al. (1997) and Gies (2003) showed that the agreement between the observed masses of massive stars in detached unevolved binaries and those determined through a comparison with evolutionary models is generally quite satisfactory for masses up to $\sim 57 \mathrm{M}_{\odot}$, provided that the 'spectral type - effective temperature' calibration of Martins et al. (2002) is adopted. Martins et al. found that the inclusion of line blanketing leads to a somewhat cooler effective temperature scale than previously suggested by Vacca et al. (1996). Consequently, the bolometric luminosities and the masses determined by comparison with evolutionary tracks are also reduced.

Finally, one has to bear in mind that the detailed comparison with evolutionary tracks may require an additional parameter to be considered. Meynet \& Maeder (2000) pointed out that rotation can have a significant impact on the evolutionary track of a massive star in the Hertzsprung-Russell diagram (see also the contributions by A. Maeder in this volume). For low gravity objects that evolved from rapidely rotating progenitors, the comparison between models with and without rotation can lead to relative mass differences of order $30 \%$, the inclusion of rotation leading to lower masses for a given luminosity.

\subsection{Massive binaries in the young open cluster Trumpler 16}

The very young open cluster Trumpler 16 (Massey \& Johnson 1993) in the Carina complex is particularly rich in massive binaries (Levato et al. 1991). For instance, this cluster harbors the only known galactic binary containing an unevolved star of spectral type O3 V (HD 93205; recently reclassified as O3.5 V $\left(\left(\mathrm{f}^{+}\right)\right)$by Walborn et al. 2002; see also below). On the other hand, the cluster contains also at least two eclipsing binaries (Tr 16-1 and $\operatorname{Tr} 16-104)$. In this section, we will briefly review the properties of these objects and the information they provide on the parameters of massive stars and on the properties of the open cluster itself. 


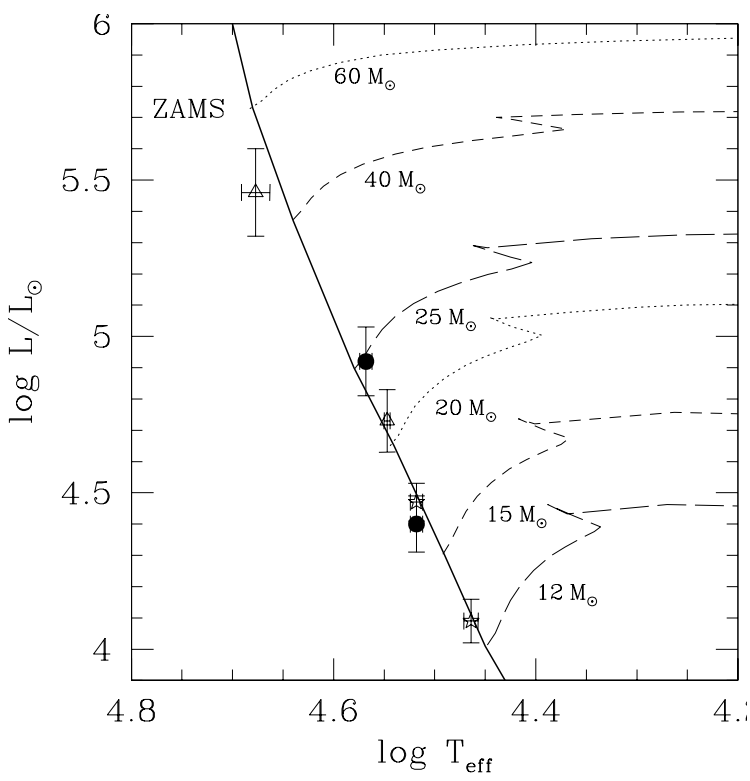

Fig. 1. Hertzsprung-Russell diagram of eclipsing or ellipsoidal early-type binaries in the open cluster Tr 16 of the Carina complex. The evolutionary tracks are taken from Schaller et al. (1992) for solar metallicity. The different symbols stand for HD 93205 (triangles), Tr 16-104 (filled dots) and Tr 16-1 (stars).

We have displayed the properties of the components of the eclisping binaries Tr 16-1 (Freyhammer et al. 2001) and Tr 16-104 (Rauw et al. 2001a) as well as of the ellipsoidal variable HD 93205 (Morrell et al. 2001, Antokhina et al. 2000) in a Hertzsprung-Russell diagram. We adopted the radii determined from the photometric analyses as well as the temperature scale proposed by Martins et al. (2002). In Fig. 1, we have also drawn the evolutionary tracks corresponding to theoretical models from Schaller et al. (1992) for solar metallicity. The components of the three binaries are all found to lie pretty close to the zero-age main sequence (ZAMS). This result suggests that $\operatorname{Tr} 16$ contains stars that are probably younger than 1 million years.

Adopting the bolometric corrections from Humphreys \& McElroy (1984), we can use the bolometric magnitudes directly obtained from the light curve analyses to compute the absolute magnitudes $\mathrm{M}_{\mathrm{V}}$. The results are given in the sixth column of Table 1, whilst the last column of this table provides the absolute visible magnitudes for stars of this spectral type following the calibration of Humphreys \& McElroy (1984). It becomes immediatly clear that the stars on the ZAMS in Tr 16 are about one magnitude fainter than expected from the Humphreys \& McElroy calibration. This discrepancy is further enhanced if we compare our observed absolute magnitudes with the absolute magnitudes proposed by Vacca et al. (1996). 
Table 1. Properties of three short-period eclipsing or ellipsoidal early-type binaries in the young open cluster Trumpler 16. The effective temperatures are based on the calibration of Martins et al. (2002). The last column provides the absolute visual magnitudes according to the calibration of Humphreys \& McElroy (1984).

\begin{tabular}{|c|c|c|c|c|c|c|}
\hline \multicolumn{2}{|l|}{ System } & $\mathrm{R} / \mathrm{R}_{\odot}$ & $\mathrm{T}_{\mathrm{eff}}(\mathrm{K})$ & $\log \mathrm{L} / \mathrm{L}_{\odot}$ & $\mathrm{M}_{\mathrm{V}, \mathrm{obs}}$ & $\mathrm{M}_{\mathrm{V}, \mathrm{cal}}$ \\
\hline HD 93205 & $\mathrm{O} 3.5 \mathrm{~V}$ & $8.0 \pm 1.2$ & $47500 \pm 1500$ & $5.46 \pm 0.14$ & -4.60 & -5.50 \\
\hline П1 90200 & O8V & $6.3 \pm 0.7$ & $35250 \pm 250$ & $4.73 \pm 0.10$ & -3.58 & -4.80 \\
\hline $\operatorname{Tr} 16-104$ & $\begin{array}{l}\mathrm{O} 7 \mathrm{~V} \\
\mathrm{O} 9.5 \mathrm{~V}\end{array}$ & $\begin{array}{l}7.1 \pm 0.9 \\
4.9 \pm 0.5\end{array}$ & $\begin{array}{l}37000 \pm 500 \\
33000 \pm 500\end{array}$ & $\begin{array}{l}4.92 \pm 0.11 \\
4.40 \pm 0.09\end{array}$ & $\begin{array}{l}-3.95 \\
-3.00\end{array}$ & $\begin{array}{l}-5.00 \\
-4.20\end{array}$ \\
\hline $\operatorname{Tr} 16-1$ & $\begin{array}{l}\mathrm{O} 9.5 \mathrm{~V} \\
\mathrm{~B} 0.3 \mathrm{~V}\end{array}$ & $\begin{array}{l}5.3 \pm 0.3 \\
4.4 \pm 0.3\end{array}$ & $\begin{array}{l}33000 \pm 500 \\
29100 \pm 500\end{array}$ & $\begin{array}{l}4.47 \pm 0.06 \\
4.09 \pm 0.07\end{array}$ & $\begin{array}{l}-3.18 \\
-2.58\end{array}$ & $\begin{array}{l}-4.20 \\
-3.80\end{array}$ \\
\hline
\end{tabular}

The reason for this discrepancy resides essentially in the stellar radii obtained from the analyses of the light curves. These radii are significantly smaller than what is usually expected for main sequence early-type stars. This result is reminiscent of the finding of Walborn \& Blades (1997) who discovered a population of subluminous, so-called $\mathrm{O} \mathrm{Vz}$, stars among the youngest objects of the 30 Doradus complex in the Large Magellanic Cloud (LMC). Walborn \& Blades suggested that these objects are nearer to the ZAMS than more luminous 'typical' O V stars.

We can use the absolute magnitudes determined from the study of the eclipsing binaries to constrain the distance of the cluster. In fact, comparing the absolute magnitudes to the apparent dereddened magnitudes, we obtain a distance modulus of $11.95 \pm 0.06$ (corresponding to a distance of $2.45 \mathrm{kpc}$ ). This value is smaller than the one determined by Massey \& Johnson (1993; $3.2 \mathrm{kpc}$ ). Again, this discrepancy may be accounted for by the fact that the O V stars in $\operatorname{Tr} 16$ are subluminous.

As stated above, Tr 16 is extremely rich in massive binaries. Several of these binaries actually belong to multiple (triple, quadruple,...) systems, some of which might be genuine hierarchical systems (e.g. Tr 16-104, Rauw et al. 2001a), while others might be line-of-sight associations (this could be the case of $\operatorname{Tr} 16-110$, Albacete-Colombo et al. 2002). Tr 16-104 consists of an eclipsing SB2 binary of period 2.15 days and a more distant third component that probably describes a longer period orbit of either 285 or 1340 days around the center of mass of the system. Tr 16-110, on the other hand, consists of an SB2 $\left(P_{\text {orb }}=3.63\right.$ days $)$ and an SB1 $\left(P_{\text {orb }}=5.03\right.$ days $)$. However the physical link between the SB2 and the SB1 is not established yet. The genuine multiple systems offer a unique opportunity to test the theory of dynamical interaction in young open clusters as well as to improve our understanding of the formation of massive stars and of the conditions of stability of hierarchical systems.

Another interesting question to address in an open cluster that rich in earlytype binaries is to determine the cut-off period for eccentric orbits that is defined as the critical period below which the tidal forces have circularised the orbit of an initially eccentric system. The existing data on binaries in $\operatorname{Tr} 16$ suggest that orbits 


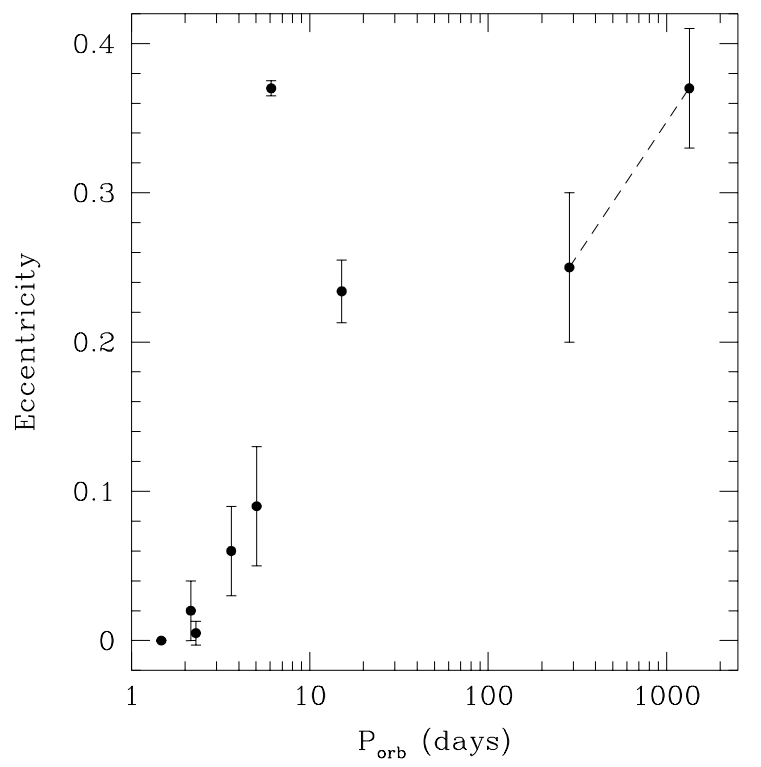

Fig. 2. Distribution of the orbital eccentricity of a sample of early-type binaries in the open cluster Tr 16 as a fonction of their orbital period. The symbols connected by the dashed line refer to the two possible SB1 orbital solutions of the third component in $\operatorname{Tr} 16-104$.

with periods below 2.5 days are indeed circular, whereas eccentricities significant at the $\geq 2 \sigma$ level are found for periods longer than 3.6 days. The fact that the tidal forces did not have enough time yet to significantly shape the $\left(\log P_{\mathrm{orb}}, e\right)$ diagram is yet another indication of the extreme youth of the stars in this cluster.

\subsection{What are the most massive stars known to date?}

An important question in the context of the formation and the stability of very massive stars is that of the mass of the most massive stars. A priori, we would expect to find them among the unevolved objects of spectral type $\mathrm{O} 2 \mathrm{~V}-\mathrm{O} 3 \mathrm{~V}$ (Walborn et al. 2002). Indeed, the evolution of massive objects is largely influenced by their huge mass loss rates and it is therefore expected that they have lost a sizeable fraction of their mass once they reach more advanced evolutionary stages (such as the WN or WC stage).

From a comparison with evolutionary tracks, Walborn et al. (2002) infer masses of up to $\sim 200 \mathrm{M}_{\odot}$ for the most luminous O2 stars (Pismis 24-1, Cyg OB2 22A). Do stars that massive really exist? Let us consider the most massive binary systems known so far. First of all, we should point out that, to date, there is no known binary system that harbors an $\mathrm{O} 2$-star. One has to bear in mind that the binarity 
of such a system might remain undetected if the mass ratio (primary/secondary) were large. In fact, the large mass of the $\mathrm{O} 2$ primary would then lead to a low amplitude of its radial velocity curve and a large mass ratio would also imply a large luminosity ratio, such that the spectral signature of the secondary in the composite spectrum would be extremely weak.

The lack of $\mathrm{O} 2$ binaries is even more embarrassing considering that the evolutionary masses of $\mathrm{O} 2 \mathrm{~V}$ - O3 V stars determined by Walborn et al. span an extremely wide range from about 50 to $200 \mathrm{M}_{\odot}$ ! Therefore, even if we knew a binary system harboring an $\mathrm{O} 2$ star with a mass below say $100 \mathrm{M}_{\odot}$, we would not be able to draw a general conclusion about the masses of the stars in this spectral class.

Over the last years, the search for the most massive star in a binary system focused on HD 93205, the O3.5 V + O8 V system discussed hereabove. HD 93205 is indeed the only known binary system in our Galaxy that contains such an early main-sequence star. As indicated in Section 2.2, this system consists of two stars that are still on the ZAMS. The orbital solution of Morrell et al. (2001) yields a mass ratio of 2.4. Assuming a typical mass of $22-25 \mathrm{M}_{\odot}$ for the $\mathrm{O} 8 \mathrm{~V}$ secondary, the mass of the O3.5 V primary would 'only' be of the order of 52 $60 \mathrm{M}_{\odot}$. A fit of the ellipsoidal light variations (amplitude $0.02 \mathrm{mag}$ ) yields a 'bestfit' orbital inclination of $60^{\circ}$ (although the uncertainties on this value are quite large; see Antokhina et al. 2000) corresponding to masses of 48 and $20 \mathrm{M}_{\odot}$ for the $\mathrm{O} 3.5 \mathrm{~V}$ and $\mathrm{O} 8 \mathrm{~V}$ component respectively. Therefore, the mass of the primary of HD 93205 appears rather 'modest'! What about binaries harboring O3 V stars in other galaxies? Interestingly, there are indeed at least three eclipsing binaries harboring very massive O V stars in the $\mathrm{R} 136$ cluster of the 30 Dor complex in the LMC (Massey et al. 2002). The extreme youth of R 136 suggests that these stars are representative of the population of O3 stars on the ZAMS (although at a lower than solar metallicity). The remarkable analysis of Massey et al. indicates that the binaries R 136-38 (O3 V + O6 V) and R 136-42 (O3 V + O3 V) have primaries with masses below $57 \mathrm{M}_{\odot}$, again far from the $200 \mathrm{M}_{\odot}$ suspected for some $\mathrm{O} 2$ stars.

Although we argued above that the most massive stars are to be found among the ZAMS objects, let us nevertheless consider binary systems in more advanced evolutionary stages. For a long time, Plaskett's Star (HD 47129) was thought to be the best candidate for the heavy-weight record-holder. This O7.5 I + O6 I binary with a 14.4 day orbital period consists of two very massive stars whose masses have been estimated to be of the order of 42.5 and $51.0 \mathrm{M}_{\odot}$ for the primary and secondary respectively (Bagnuolo et al. 1992). However, these results must be considered with caution since HD 47129 does not display photometric eclipses allowing an accurate orbital inclination to be determined.

Another extremely massive object is the WN7ha primary in the 80-day period binary WR 22. Rauw et al. (1996) derived a minimum mass of $72 \mathrm{M}_{\odot}$ for this star. Since the system displays atmospheric eclipses when the WNha star passes in front of its secondary (Gosset et al. 1991), the orbital inclination is pretty close to $90^{\circ}$ (Rauw 1997) and the actual mass should be close to the minimum mass. However, Schweickhardt et al. (1999) obtained a more extensive data set of this 
system and found a somewhat larger orbital eccentricity than Rauw et al. (1996) leading to a downwards revision of the mass of the primary (although with a mass of $55 \mathrm{M}_{\odot}$ it remains one of the most massive stars known in our Galaxy).

In summary, it appears that so far there is hardly any evidence that stars with masses exceeding $60 \mathrm{M}_{\odot}$ actually exist in binary systems. Although this does not rule out the existence of stars with larger masses, high-quality radial velocity studies and photometric monitoring of the candidates for these extremely massive objects are urgently needed to investigate their multiplicity and uncover possible binary systems. An important question to address from the theoretical point of view would be whether there could exist a difference in the upper mass limit for the formation of single and binary massive stars.

\subsection{Observational evidence for mass exchange in evolved massive binaries?}

As stated above the results from the study of early-type binaries can be used to infer the properties of single massive stars only if the binary system has not suffered from any mass exchange. However, many massive binaries are found to be evolved systems. In some cases, the comparison of the properties of their components with evolutionary tracks for single massive stars yields inconsistent results. For instance, in the case of HD 152248 (O7.5 III(f) + O7 III(f)) both stars appear overluminous for their masses (Sana et al. 2001) and the evolutionary masses needed to account for the observed luminosity are $\sim 30 \%$ larger than the observed masses. Penny et al. (1996) argued that this behaviour is an indication of ongoing Roche lobe overflow (RLOF). We note however, that the available light curve solution of HD 152248 (Mayer et al. 2001) does not support this idea. The present-day configuration of HD 152248 may have been affected by a past RLOF episode instead (Sana et al. 2001). On the other hand, as stated previously the inclusion of rotation in the single star evolutionary models may in fact reduce the discrepancy between the observed and theoretical masses. However, in this system, the observed present-day rotational velocities are not exceptionally large and it remains to be seen whether they are sufficient to account for a large effect on the evolution.

As a second example, we consider the evolved binary HDE 228766. Rauw et al. (2002b) found that this system consists of an O7 primary and a WN8ha secondary. Although the secondary is nowadays the less massive star it has clearly the stronger stellar wind and is the most evolved component of the system (as witnessed also by the peculiar chemical abundances in its wind). The secondary star appears therefore as a kind of transition object at the beginning of the WN stage and it must have been the initially more massive component of the system. The easiest way to account for this situation is to assume that the evolution of the whole system has been influenced by mass exchange. Evolutionary tracks from Vanbeveren \& de Loore (1994) and de Loore \& Vanbeveren (1994) could indeed explain the overluminosity of the secondary. The initially more massive component could have undergone mass loss through Roche lobe overflow and would now appear as the less massive star. Rauw et al. (2002b) pointed out that the 


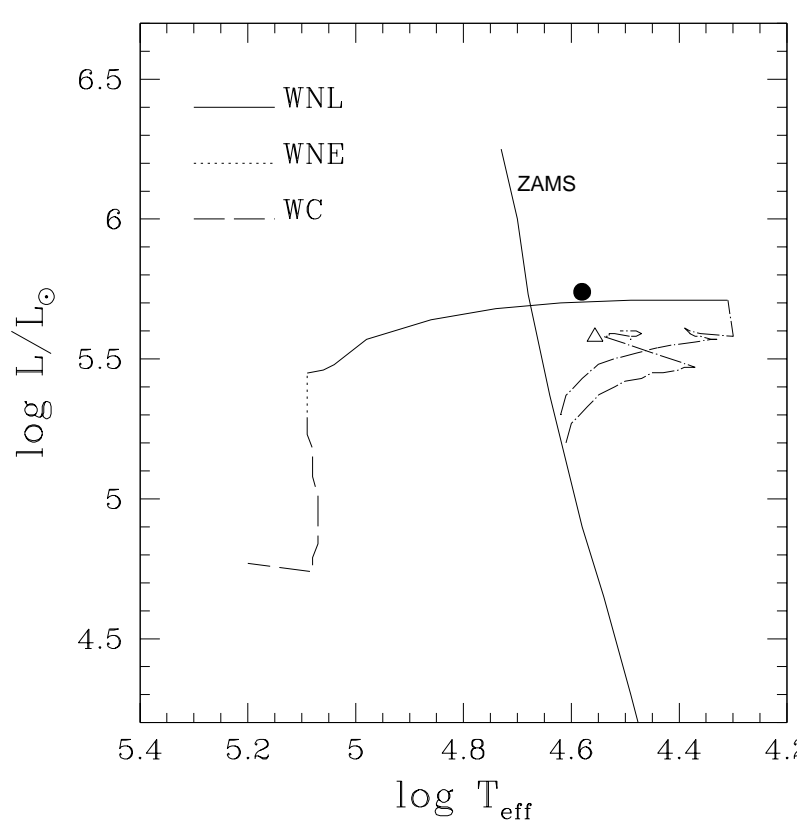

Fig. 3. Comparison of the location of the components of HDE 228766 in the HertzsprungRussell diagram with the binary evolution tracks from Vanbeveren \& de Loore (1994) and de Loore \& Vanbeveren (1994) for a binary of initial masses $40 \mathrm{M}_{\odot}+36 \mathrm{M}_{\odot}$ and an initial orbital period of 25 days. The open triangle stands for the present-day primary whereas the filled dot stands for the present-day secondary. Note that the dash-dotted tracks correspond to the evolution during the main-sequence lifetime.

primary has broader absorption lines and seems therefore to be rotating faster than the secondary. In the framework of a binary evolution model, the larger $v \sin i$ of the present-day primary could be due to spinning up through accretion (Vanbeveren \& de Loore 1994). Let us emphasize that according to the binary evolution tracks from Vanbeveren \& de Loore and de Loore \& Vanbeveren (1994) for a binary of initial masses $40 \mathrm{M}_{\odot}+36 \mathrm{M}_{\odot}$ and an initial orbital period of 25 days, the present-day parameters of both stars in HDE 228766 are consistent with an age of about 5.5 million years. It seems therefore plausible that HDE 228766 is in a post RLOF stage.

\section{Interacting winds in early-type binaries}

Early-type stars have energetic highly supersonic winds. Whenever two early-type stars find themselves bound by gravitation in a binary system, their winds interact. The wind interaction region is limited by two hydrodynamical shocks, each facing one of the binary components. The compressed stellar winds are separated by a 
contact discontinuity which is set by the equilibrium of the stellar wind momenta.

Colliding stellar winds in massive binaries provide an astrophysical laboratory to study the shock physics of cosmic plasmas. The theoretical concept of colliding stellar winds was put forward by Prilutskii \& Usov (1976) and Cherepashchuk (1976). The first models based on a numerical solution of the differential equations of the hydrodynamics governing the phenomenon were presented by Lebedev \& Myasnikov (1988), Luo et al. (1990) and Stevens et al. (1992). Although the details of the process are quite complex, some insight into the geometry and the physics of the phenomenon can be gained from rather simple considerations. Below, we will briefly introduce some theoretical aspects of colliding winds. To this aim, we closely follow the developments presented by Stevens et al. (1992).

\subsection{Some basic theoretical concepts}

Consider a system formed by two stars of radii $R_{1}$ and $R_{2}$. We assume that the stellar winds of both components are spherically symmetric and that the wind velocity at radius $r$ can be described by a so-called $\beta$-law:

$$
v_{j}(r)=v_{\infty, j}\left(1-\frac{R_{j}}{r}\right)^{\beta_{j}}, j=1,2
$$

where $v_{\infty, j}$ and $R_{j}$ stand respectively for the asymptotic wind velocity and the radius of star $j$. Each wind carries a mass loss rate $\dot{\mathrm{M}}_{j}$. The equation of the contact discontinuity follows from the condition of ram pressure equilibrium:

$$
\rho_{1}\left(r_{1}\right) v_{1}^{2}\left(r_{1}\right) \cos ^{2} \phi_{1}=\rho_{2}\left(r_{2}\right) v_{2}^{2}\left(r_{2}\right) \cos ^{2} \phi_{2}
$$

(see Fig. 4) where $\rho_{j}\left(r_{j}\right)$ stands for the density of the stellar wind of star $j$ at radius $r_{j}$. This can be written

$$
\frac{\dot{\mathrm{M}}_{1} v_{1}(r)}{4 \pi r_{1}^{2}} \cos ^{2} \phi_{1}=\frac{\dot{\mathrm{M}}_{2} v_{2}(r)}{4 \pi r_{2}^{2}} \cos ^{2} \phi_{2}
$$

Introducing the wind momentum ratio

$$
\mathcal{R}=\left(\frac{\dot{\mathrm{M}}_{1} v_{\infty, 1}}{\dot{\mathrm{M}}_{2} v_{\infty, 2}}\right)^{1 / 2}
$$

one obtains that

$$
\frac{r_{1}}{r_{2}}=\lambda \frac{\tan \beta \cos \theta_{1}-\sin \theta_{1}}{\sin \theta_{2}-\tan \beta \cos \theta_{2}}
$$

where $\lambda=\mathcal{R}\left(1-R_{1} / r_{1}\right)^{\beta_{1} / 2} /\left(1-R_{2} / r_{2}\right)^{\beta_{2} / 2}$.

Finally, after some basic algebra, we obtain the differential equation describing the contact discontinuity:

$$
\frac{d z}{d x}=\tan \beta=\frac{\left(\lambda r_{2}^{2}+r_{1}^{2}\right) z}{\lambda r_{2}^{2} x+r_{1}^{2}(x-d)}
$$




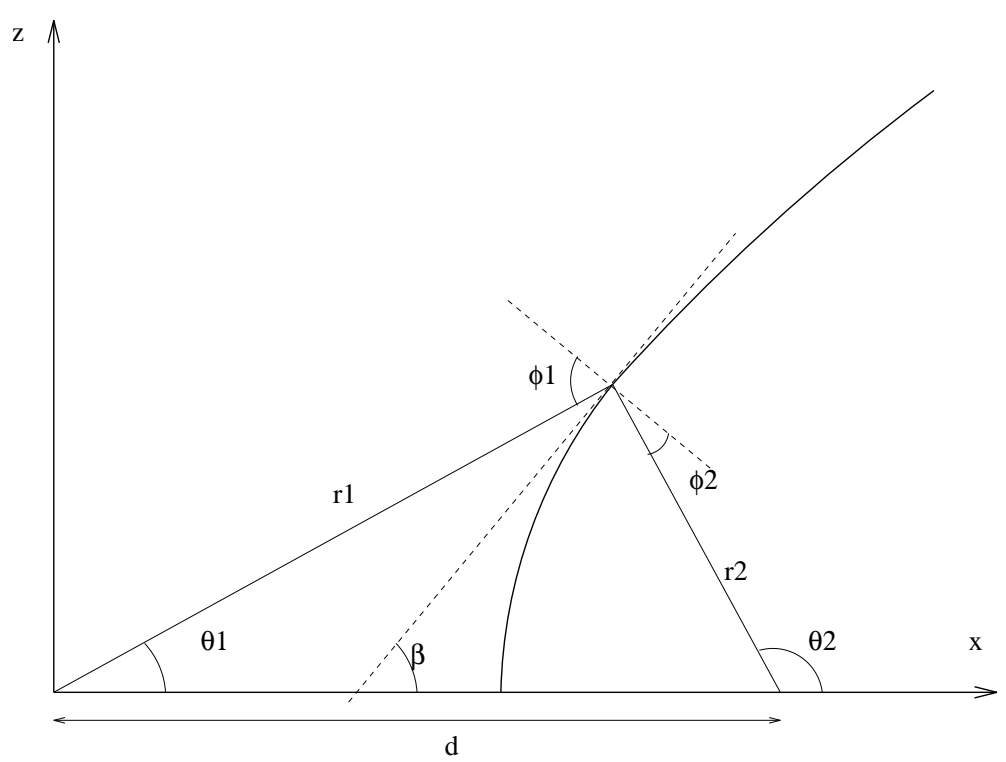

Fig. 4. Schematic view of the contact discontinuity in a colliding wind binary. The star with the more energetic wind is located at the origin of the axes, while the companion is located at a distance $d$ on the $x$-axis. We assume that the shock region has an axisymmetric shape around the binary axis.

where $r_{1}=\sqrt{x^{2}+z^{2}}, r_{2}=\sqrt{(x-d)^{2}+z^{2}}$ and $d$ stands for the distance between the centers of the stars (see Stevens et al. 1992). The shape of the contact discontinuity is then obtained through numerical integration of equation 3.3.

If both winds have reached their terminal velocity before they collide, we get $\lambda=\mathcal{R}$. In this case, the intersection between the binary axis and the surface discontinuity will be found at a distance $x^{\text {stag }}=\mathcal{R} d /(\mathcal{R}+1)$ from the origin (i.e. from the center of mass of the star with the more energetic wind). In a binary system where both winds are of equal strength $(\mathcal{R}=1)$, the contact discontinuity can be approximated by a plane located mid-way between the two stars. In a more general situation with $\mathcal{R} \neq 1$, the contact discontinuity appears as a cone wrapped around the star with the less energetic wind. The half opening angle of this shock cone may be approximated by $\Theta\left(^{\circ}\right) \sim 120\left(1-\frac{\mathcal{R}^{-4 / 5}}{4}\right) \mathcal{R}^{-2 / 3}$ (Eichler \& Usov 1993).

The immediate post-shock properties of the plasma can be described by the Rankine-Hugoniot conditions for strong shocks in a gas with $\gamma=5 / 3: \rho_{s, j}=4 \rho_{j}$, $v_{s, j}=v_{j} / 4$ and $T_{s, j}=\frac{3 \bar{m}_{j} v_{j}^{2}}{16 k}$, where $\bar{m}_{j}$ is the average mass per particle in the wind of star $j$ (see e.g. Stevens et al. 1992). For solar abundances, we find that $k T_{s, j} \simeq 1.17 v_{8}^{2}$ (in $\mathrm{keV}$ ) where $v_{8}$ is the pre-shock velocity expressed in $1000 \mathrm{~km} \mathrm{~s}^{-1}$. Therefore, due to the large pre-shock wind velocities, the post-shock 
plasma temperature is very high $\left(\geq 10^{7} \mathrm{~K}\right)$. Thus the main signature of this shockheated plasma is expected to occur in the X-ray domain.

The post-shock gas near the axis will be subsonic (due to the high post-shock temperature and the fact that the sound speed increases as $\left.\sqrt{T_{s}}\right)$. The post-shock gas flows away from the axis at velocities close to the sound speed. The properties of the post-shock gas depend to a large extent on the importance of radiative cooling that can be roughly estimated from the ratio between the characteristic cooling time and the typical escape time from the shock region:

$$
\chi=\frac{t_{\mathrm{cool}}}{t_{\mathrm{esc}}} \simeq \frac{v_{8}^{4} x_{12}^{\mathrm{stag}}}{\dot{\mathrm{M}}_{-7}}
$$

where $x_{12}^{\text {stag }}$ and $\dot{\mathrm{M}}_{-7}$ are respectively the distance to the contact surface in units $10^{7} \mathrm{~km}$ and the mass loss rate in units $10^{-7} \mathrm{M}_{\odot} \mathrm{yr}^{-1}$ (Stevens et al. 1992). If radiative cooling is important $(\chi \ll 1)$, the entire thermal energy produced in the shock is radiated immediately away. If the shock is highly radiative, it collapses and the ionization of the material in the interaction region is set by the radiation of the stars rather than by the shock. This rather cool material can contribute to the formation of emission lines in the optical spectrum. Otherwise, if $\chi \geq 1$, the shock region is adiabatic. From the $\chi$ parameter defined by Stevens et al. (1992), it becomes immediately clear that the larger the mass loss rate (all other things being equal), the more efficient the cooling.

The shocked winds are seldom stable. Different kinds of instabilities may affect the material in the interaction region and these phenomena are usually more pronounced for radiative winds.

We ought to mention that the shocks associated with a wind collision can act as sites for particle acceleration through the first order Fermi mechanism (Eichler \& Usov 1993). In this scenario free electrons undergo acceleration to relativistic velocities by crossing the shock front forth and back (see e.g. Longair 1992).

Over the last ten years it became clear that there are several effects that may substantially affect the predictions of the numerical simulations. The most prominent ones are:

- deviations of the wind velocity law from the standard $\beta$-law description. There are various reasons why the $\beta$-law may not be a good approximation especially in close binary systems: radiative inhibition (Stevens \& Pollock 1994) and radiative braking (Gayley et al. 1997) are the best understood effects. In addition, X-ray emission from the shock region may also alter the ionization of the wind material and therefore affect its ability to suffer radiative acceleration.

- 3-D effects that are often neglected in the numerical models. The Coriolis force may have an important impact on the shape of the interaction region if the orbital period is short enough for the orbital velocities to become significant compared to the wind velocities (see e.g. Pittard 2000). 


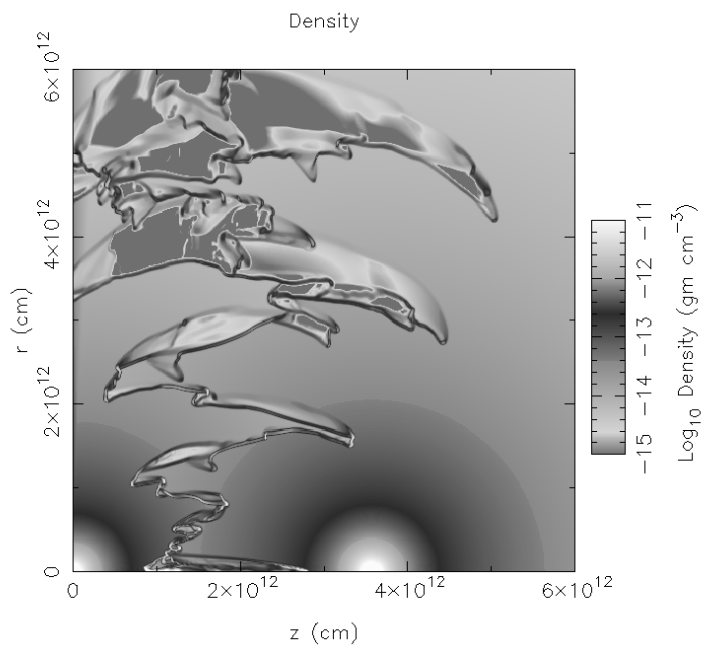

Fig. 5. Snapshot grey-scale images of the density structure of a hydrodynamical simulation of a highly radiative wind collision. The $z$-axis corresponds to the line of centers whilst $r$ yields the radial distance from this axis. The wind parameters are such that $\mathcal{R}=1$ and $\chi \simeq 0.07$ for the shocked winds of both stars (courtesy Sana et al. 2003, in preparation).

\subsection{Observational evidence for colliding winds}

\subsubsection{In the optical and UV domain...}

Optical and ultraviolet spectra of many early-type binaries display emission line profile variability. Examples of evolved $\mathrm{O}+\mathrm{O}$ star binaries that show such phaselocked profile variations are AO Cas (O9 III + O8 V; Gies et al. 1997), 29 CMa (O7.5-8 Iabf + O9.7 Ib; Wiggs \& Gies 1993), Plaskett's Star (HD 47129, O7.5 I + O6 I, Wiggs \& Gies 1992), HD 93403 (O5.5 I + O7V; Rauw et al. 2002a), HD 149404 (O7.5I(f) + ON9.7 I; Rauw et al. 2001b), HD 152248 (O7.5 III(f) + O7 III(f); Sana et al. 2001), V 729 Cyg (O6-7 Ia + Ofpe/WN9; Rauw et al. 1999)... Examples of colliding wind WR binaries showing emission line profile variations are HD 5980 (WN? + ?; Breysacher \& François 2000), WR 30a (Gosset et al. 2001), WR 42 (WC7 + O; Hill et al. 2000), WR 79 (WC7 + O; Hill et al. 2000), WR 139 (WN5 + O6 III-V, Marchenko et al. 1997)...

Many of the WR + O systems (especially the $\mathrm{WC}+\mathrm{O}$ ) display moving subpeaks on top of flat-topped broad emission lines. The line profile variability reflects the loss of spherical symmetry of the winds. In fact, the variations can be due either to geometrical effects produced by the cavity in the emitting/absorbing wind volume or to excess emission coming from the high density material compressed in the wind interaction region. In a highly radiative shock, the material in the interaction region cools sufficiently to reach temperatures where optical and UV 


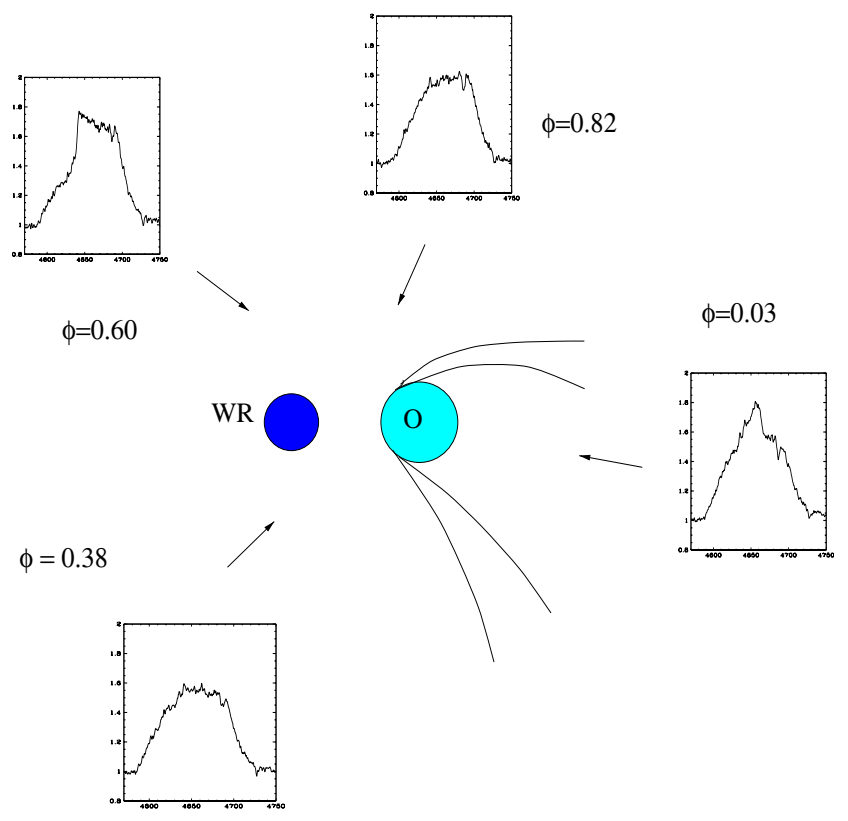

Fig. 6. Orbital variability of the CIV $\lambda 4658$ blend in the spectrum of WR 30a. The arrows indicate the projections of the line of sight on the orbital plane at various phases, while the insets display the C IV profiles observed at these phases (see Gosset et al. 2001).

recombination lines are formed. Due to the density squared dependence of the emissivity of the recombination process, the high-density material in the postshock region can contribute significantly to the total line emission spectrum of an early-type binary.

To illustrate this phenomenon, we consider the variations of the CIV $\lambda 4658$ blend in the spectrum of the WO4 + O5((f)) binary WR30a (Gosset et al. 2001). Two time-series of spectra taken one year apart revealed identical line profile variations clearly demonstrating the phase-locked nature of the phenomenon. The variations result from an apparent extra emission on top of the broad line (see Fig. 6). The phase dependence of this feature is consistent with excess emission produced mainly in the trailing arm of the shock region. Due to the Coriolis effect, the shock cone is probably deflected by about $18^{\circ}$ and the emissivity of the wind material is most likely larger in the trailing wake where the shock of the WR wind is more head-on.

Although the most straightforward explanation for the observations related to phase-locked line profile variability in the optical spectra of early-type binaries is indeed a colliding wind scenario, present day hydrodynamic simulations fail to reproduce this feature. The main reason for this failure is the fact that the numerical grids undersample the cooling layers of the shock region. 


\subsubsection{In the radio domain...}

A subset of early-type stars display a non-thermal radio emission in addition to the thermal radio emission produced via free-free emission from the stellar wind. The non-thermal (synchrotron) emission is a definite sign of two things: (i) a magnetic field must be present in the winds of these objects and (ii) relativistic electrons exist in the radio emitting region. As stated above, the relativistic electrons may be accelerated through the first order Fermi mechanism in the shock zone of the wind interaction region (Eichler \& Usov 1993). Interestingly, many of the non-thermal radio emitting early-type stars are binary systems. For instance, Dougherty \& Williams (2000) pointed out that at least seven out of nine WR stars that display non-thermal radio emission are in fact binaries.

The most famous examples in this category are probably WR 140 (WC7 + O5, e.g. White \& Becker 1995) and WR 147 (e.g. Williams et al. 1997).

WR 140 has a highly eccentric $(e=0.84)$ orbit with a period of $7.9 \mathrm{yrs}$ and the radio flux displays a phase-locked variation (White \& Becker 1995). The radio flux is thermal over large parts of the orbit. However, between phases 0.55 and 0.95 (phase 0.0 corresponding to the periastron passage), the radio flux increases dramatically and displays a non-thermal spectral index. The shape of the radio light curve at different frequencies results basically from a combination of two effects. First, during most of the orbit, the two stars are so close that the region of the wind interaction is burried deep inside the (huge) radio photosphere of the WR star and therefore non-thermal radio emission produced by electrons accelerated in the shock can only be observed at phases near apastron when the shock zone is farthest away from the WR core and moves out of the WR's radio photosphere (remember that the distance of the shock from the WR star, $x^{\text {stag }}$ scales with the binary separation $d$ ). Second, as a result of the cavity produced by the shock cone in the WR wind, the non-thermal radio emission can reach us when the O-star with its lower density (and thus less opaque) wind passes in front of the WR star.

WR 147 is a visual binary consisting of a WN8 star and a late-O, early-B type star. The radio emission from this system was resolved by MERLIN into two components: a thermal component associated with the wind of the WN8 star and a non-thermal component located in between the WR star and its visual companion (but closer to the latter, see Williams et al. 1997). The location of this nonthermal component is consistent with the ram pressure balance of the two stellar winds as expected in a colliding wind scenario. Interestingly, WR 147 is also the first early-type system where Chandra ACIS-I observations revealed evidence for a spatially extended X-ray emission. The X-rays are found to peak close to the position of the radio bow shock (Pittard et al. 2002).

\subsubsection{In the infrared...}

Some WC stars (some of which are known binaries usually with wide orbital separations) exhibit episodic variability of their IR emission. At least in two cases (WR 140 and WR 137) this phenomenon is recurrent. These variations have been attributed to dust formation in the wind collision zone (see Williams 2002 for a 
review). However, this wind interaction zone offers a rather hostile environment for dust to form. Although the chemical ingredients to make dust are available in a WC wind, the intense radiation from the WR stars and its O-type companion should prevent dust from forming. Even more critical is the fact that the temperature in the shocked winds of a binary with an adiabatic wind collision is much higher than what is required for dust to form and it remains to be seen whether the density $\left(\geq 10^{12} \mathrm{~cm}^{-3}\right)$ and temperature $(\leq 3900 \mathrm{~K})$ conditions required to initiate dust formation (Le Teuff 2002) can be met in the wind interaction zone. Recent hydrodynamical simulations suggest that clumpiness of the WR wind might help to overcome these problems (see Walder \& Folini 2003).

At least in the case of WR 140, the IR outbursts (variation by 3 magnitudes at $3.8 \mu \mathrm{m}$ ) appear to be phase-locked. The outburst events are initiated a few months after periastron passage, indicating that material is swept away from the interaction region, only forming dust at some distance downstream (Williams 2002).

Another, probably related observation is the detection of pinwheel nebulae associated with several WC binaries. In fact, observing with the Keck I telescope in the near infrared, Tuthill et al. (1999) discovered that WR 104 displays a spiral shaped nebula that is moving around the center of mass of the binary as the stars are revolving around each other once in 220 days. In this case, it seems that the infrared excess is produced in the tail of the shock region wrapped around the O-star companion. Dust produced in the wake of the O-star would be carried out of the system by the WR wind and the combination of the radial wind with the orbital motion results in a spiral-like feature. Due to the shadowing effect of the inner coil of the spiral, its outer parts are not heated by the central stars and remain therefore invisible in the near IR.

\subsubsection{In the X-rays...}

As stated above, the main signature of wind interactions, especially in the adiabatic case, is expected in the X-ray domain. However, individual early-type stars are also intrinsic X-ray emitters. To disentangle the shock emission from the intrinsic $\mathrm{X}$-ray emission, we can compare the observed $\mathrm{L}_{\mathrm{X}} / \mathrm{L}_{\mathrm{bol}}$ ratio to the 'canonical' scaling relation derived by Berghöfer et al. (1997).

On statistical grounds, early-type binaries are found to be stronger X-ray sources than their single counterparts (Pollock 1987, Chlebowski \& Garmany 1991). However, there is a considerable scatter in the $\mathrm{L}_{\mathrm{X}} / \mathrm{L}_{\mathrm{bol}}$ relation. Therefore, it is important to have an additional criterion allowing to establish the link between colliding winds and X-ray emission in early-type binaries. The X-ray emission from colliding wind binaries is expected to display a significant phaselocked modulation (Pittard \& Stevens 1997) either as a consequence of changing opacity along the line of sight or as a consequence of a changing separation between the stars in eccentric binaries (for an adiabatic shock, the X-ray luminosity is expected to scale as $\mathrm{L}_{\mathrm{X}} \propto \dot{\mathrm{M}}^{2} / d$, Stevens et al. 1992). Corcoran (1996) presented the ROSAT-PSPC light curves of sixteen O-type binaries and found evidence for a phase-locked modulation in at least four cases. 


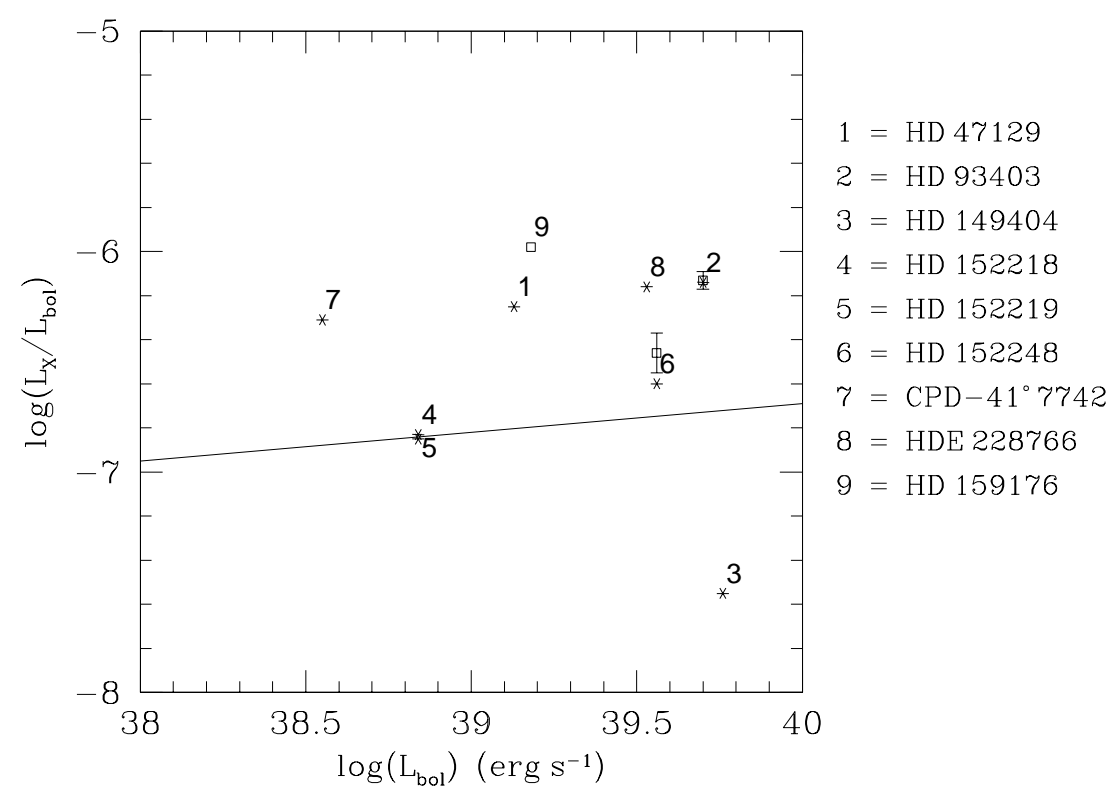

Fig. 7. ROSAT-PSPC and XMM-Newton EPIC X-ray luminosities in the energy range 0.1 to $2.0 \mathrm{keV}$ for a sample of massive close $\mathrm{O}+\mathrm{O}$ binaries. The solid line shows the empirical scaling relation derived by Berghöfer et al. (1997). Stars stand for ROSAT data whereas open squares stand for XMM data. Note the good agreement between ROSAT and XMM data for HD 93403 and HD 152248. While most of the binaries are X-ray over-luminous, two systems (HD 152218 and HD 152219) exhibit no excess emission and HD 149404 even appears underluminous. The 'error bars' on the data points of HD 93403 and HD 152248 indicate the range of orbital variability of their X-ray luminosities.

A textbook example of a $\mathrm{WR}+\mathrm{O}$ colliding wind binary is the WC8 + O7.5 III binary $\gamma^{2} \operatorname{Vel}\left(=\mathrm{WR} 11, P_{\text {orb }}=78.53\right.$ days $)$. Willis et al. (1995) gathered ROSATPSPC observations of this system that revealed substantial phase-locked variability of the X-ray flux. These authors attributed the increase of the X-ray flux between phases 0.0 and 0.2 to extra emission from a colliding wind interaction. The X-ray emission from the shock is highly absorbed when the dense and opaque WR wind is in front but becomes significantly less absorbed at orbital phases when the cavity around the O-star crosses the line of sight. Stevens et al. (1996) showed that a detailed comparison between two ASCA observations of $\gamma^{2}$ Vel and synthetic spectra calculated from hydrodynamic colliding wind models allow to constrain the mass loss rate of the WC8 component.

A number of early-type binaries have been monitored with previous X-ray satellites (for a review see e.g. van der Hucht 2002). Some very recent results have been published based on observations with the XMM-Newton and Chandra observatories. These include Chandra observations of WR 147 (Pittard et al. 2002), 


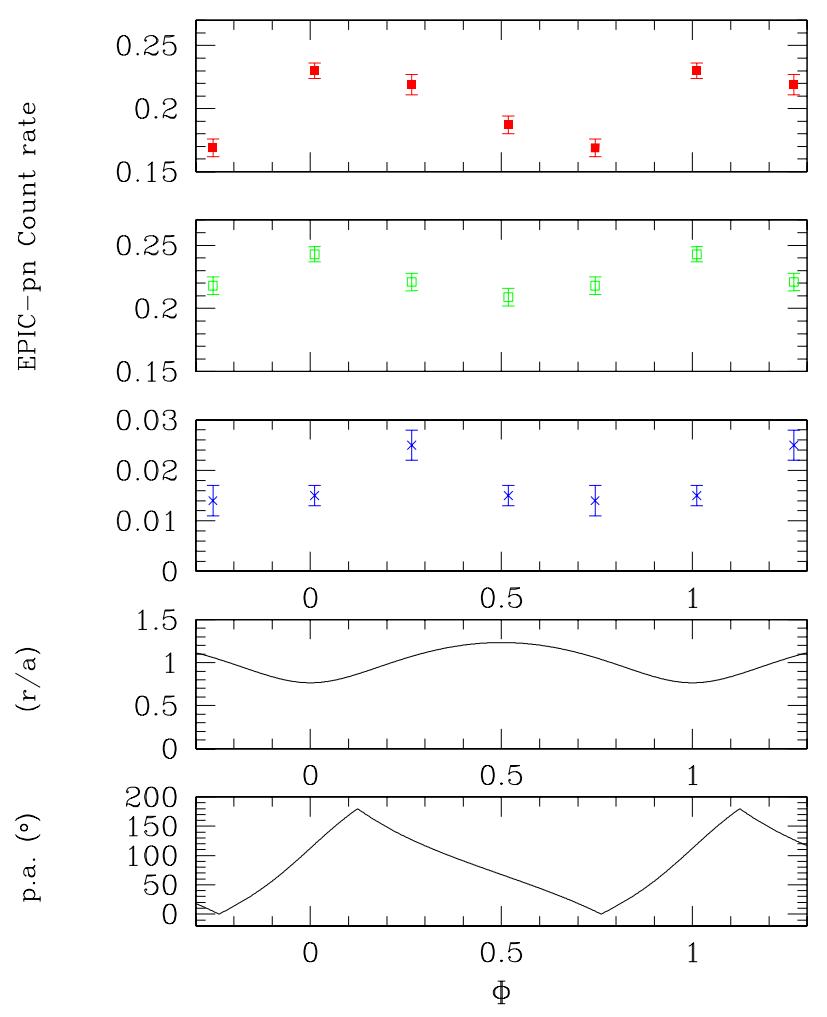

Fig. 8. XMM-Newton light curve of HD 93403. The upper, second and third panels display the EPIC-pn count rates respectively over the $0.5-1.0$ (soft), $1.0-2.5$ (medium) and $2.5-10.0 \mathrm{keV}$ (hard) energy bands. 1- $\sigma$ error bars are indicated. The fourth panel yields the relative orbital separation $(r / a)$ between the components of HD 93403 ( $a$ stands for the semi-major axis of the orbit). Finally, the lower panel provides the position angle of the binary axis. A p.a. of $0^{\circ}$ corresponds to the primary star being 'in front' of the secondary (Rauw et al. 2002a).

Chandra and XMM-Newton observations of $\gamma^{2}$ Vel (Skinner et al. 2001, Dumm et al. 2003), XMM-Newton observations of HD 93403 (Rauw et al. 2002a) and WR 22 (Gosset et al. 2003).

Rauw et al. (2002a) presented XMM-Newton observations of the O-star binary HD 93403 revealing phase-locked variability of its X-ray flux and of its optical spectrum. The colliding wind scenario offers a promising explanation for both phenomena. Below $1.0 \mathrm{keV}$, the observed X-ray flux is essentially modulated by the opacity of the primary wind. Above $1.0 \mathrm{keV}$, the observed variation of the $\mathrm{X}$-ray flux is roughly consistent with a $1 / d$ dependence expected for an adiabatic colliding wind interaction. However, not all early-type binaries display the $1 / d$ dependence of their X-ray flux. For instance, Pittard et al. (2000) analyzed two 
ASCA observations of $\iota$ Ori obtained near periastron and apastron. Strong differences between these phases were a priori expected, but the observations proved otherwise: both the luminosity and the spectral shapes were found to be remarkably similar. This suggests that the bulk of the X-ray emission in $\iota$ Ori is in fact intrinsic to one of the components rather than being associated with the wind interaction.

Another interesting X-ray light curve is provided by the XMM-Newton observations of WR 22 (WN7ha + O6.5-8.5). In this system, the X-ray flux decreases smoothly from apastron to periastron. This behaviour is in stark contrast with a $1 / d$ variation. However, if the count rates are divided into a soft $(0.5-2.0 \mathrm{keV})$ and hard $(2.0-5.0 \mathrm{keV})$ band, it becomes clear that the modulation seen in the broad band light curve essentially results from the variations in the soft band, whereas the harder flux remains essentially constant. Since periastron passage corresponds to the atmospheric eclipse of the O-star by the WR wind (Gosset et al. 1991), it seems likely that the observed variations reflect the progressive extinction of the O-star's intrinsic X-ray emission by the opacity of the WR wind. WR 22 offers therefore the possibility to use the X-ray emission of the O-star as a probe to scan the wind opacity of the WN7ha wind.

\subsubsection{Predictions for the $\gamma$-ray domain}

Finally, we consider the situation in the $\gamma$-ray domain. Romero et al. (1999) found a significant correlation (at the $4 \sigma$ level) of unidentified EGRET sources with OB associations. Their study further provides evidence that several individual massive binaries, including WR 140 and the multiple system V 729 Cyg, are probable counterparts of $\gamma$-ray sources (see also Chen et al. 1996; Benaglia et al. 2001).

As stated above, the shocks in a colliding wind binary provide an excellent site for the acceleration of charged particles to relativistic energies (Eichler \& Usov 1993). Because of the strong UV radiation field of luminous early-type stars, relativistic electrons are expected to dissipate most of their energy through the inverse-Compton scattering mechanism (Mücke \& Pohl 2001) and the expected luminosities are of the order of a few times $10^{34} \mathrm{erg} \mathrm{s}^{-1}$ (Benaglia et al. 2001).

In addition, relativistic protons lose their energy in the interaction with thermal ions in the densest part of the stellar wind producing neutral pions that decay into $\gamma$-rays with a symmetric spectrum peaking around $70 \mathrm{MeV}$. The total luminosity due to $\pi^{0}$-decay depends on the wind density and hence the mass loss rate (the denser the wind, the more opaque it is to the ion-ion interactions). Theoretical models predict values of about $10^{33} \mathrm{erg} \mathrm{s}^{-1}$ (White \& Chen 1992).

As shown by Benaglia et al. (2001), the energy contents of the relativistic electron population in the wind interaction region of V $729 \mathrm{Cyg}$ and the local radiation fields could result in a significant $\mathrm{keV}-\mathrm{MeV}$ inverse-Compton flux from this system. Forthcoming INTEGRAL observations will allow to check these predictions and therefore provide a unique result that has been lacking for even the best-known colliding wind systems in the past. 


\section{Conclusions}

Many fundamental properties of early-type stars can be determined in a direct way through the study of binary systems. Among the most critical problems that should be addressed is the question of the multiplicity of extremely massive star candidates. An observational as well as a theoretical effort are needed to check whether extremely massive stars actually exist in binary systems.

The study of eclipsing binaries further suggests that the 'standard' luminosities of single early-type stars may be overestimated at least for very young main sequence stars. ESA's forthcoming astrometric mission GAIA will allow to establish accurate distances for most of the early-type stars known in our Galaxy. Together with an in-depth study of eclipsing binaries these results will provide constraints on bolometric corrections and other parameters that are still rather uncertain.

Our understanding of wind interactions in early-type binaries is still somewhat fragmentary. The most obvious questions deal with the formation of dust and optical emission lines respectively in adiabatic and radiative colliding wind systems. But a lot of work remains to be done on the X-ray and radio side as well. For instance, multiplicity has still to be established for some of the O or WR stars displaying non-thermal radio emission. Also a deeper understanding of the X-ray emission is needed to distinguish between excess emission from the shocked winds and intrinsic emission from the individual components.

My thanks go to the organizers of the Aussois school for their invitation to present this review and to the participants for many stimulating discussions. I wish to express my gratitude to my colleagues from the Groupe d'Astrophysique des Hautes Energies in Liège and especially to Drs. Jean-Marie Vreux and Jean-Pierre Swings who initially triggered my interest in the study of high energy phenomena associated with massive stars. I am greatly indebted to the Fonds National de la Recherche Scientifique (Belgium) for multiple assistance. This research is also supported in part by contracts P4/05 and P5/36 "Pôle d'Attraction Interuniversitaire" (SSTC-Belgium) and through PRODEX grants linked to the ESA XMM-Newton and INTEGRAL missions. 


\section{References}

Albacete-Colombo, J.F., Morrell, N.I., Rauw, G., Corcoran, M.F., Niemela, V.S., \& Sana, H. 2002, MNRAS, 336, 1099

Antokhina, E.A., Moffat, A.F.J., Antokhin, I.I., Bertrand, J.-F., \& Lamontagne, R. 2000, ApJ, 529, 463

Bagnuolo, W.G.Jr., Gies, D.R., \& Wiggs, M.S. 1992, ApJ, 385, 708

Bagnuolo, W.G.Jr., Gies, D.R., Hahula, M.E., Wiemker, R., \& Wiggs, M.S. 1994, ApJ, 423, 446

Benaglia, P., Romero, G.E., Stevens, I.R., \& Torres, D.F. 2001, A\&A, 366, 605

Berghöfer, T.W., Schmitt, J.H.M.M., Danner, R., \& Cassinelli, J.P. 1997, A\&A, 322,167

Breysacher, J., \& François, P. 2000, A\&A, 361, 231

Burkholder, V., Massey, P., \& Morrell, N. 1997, ApJ, 490, 328

Chen, W., White, R.L., \& Bertsch, D. 1996, A\&AS, 120, 423

Cherepashchuk, A.M. 1976, Sov. Ast. Lett., 2, 138

Chlebowski, T., \& Garmany, C.D. 1991, ApJ, 368, 241

Corcoran M.F. 1996, Rev. Mex. Astron. Astrofis. Conf. Series, 5, 54

de Loore, C., \& Vanbeveren, D. 1994, A\&A, 292, 463

Dougherty, S.M., \& Williams, P.M. 2000, MNRAS, 319, 1005

Dumm, T., Güdel, M., Schmutz, W., Audard, M., Schild, H., Leutenegger, M., \& van der Hucht, K.A. 2003, in "New Visions of the X-ray Universe in the XMM-Newton and Chandra Era", ed. F. Jansen, ESA SP-488, in press

Eichler, D., \& Usov, V.V. 1993, ApJ, 402, 271

Freyhammer, L.M., Clausen, J.V., Arentoft, J., \& Sterken, C. 2001, A\&A, 369, 561

Gayley, K.G., Owocki, S.P., \& Cranmer, S.R. 1997, ApJ, 475, 786

Gies, D.R. 2003, in "A Massive Star Odyssey, from Main Sequence to Supernova", Proc. IAU Symp. 212, eds. K.A. van der Hucht, A. Herrero \& C. Esteban, in press

Gies, D.R., Bagnuolo, W.G.Jr., \& Penny, L.R. 1997, ApJ, 479, 408

Gosset, E., Remy, M., Manfroid, J., Vreux, J.-M., Balona, L.A., Sterken, C., \& Franco, G.A.P. 1991, IBVS, 3571, 1

Gosset, E., Royer, P., Rauw, G., Manfroid, J., \& Vreux, J.-M. 2001, MNRAS, 327, 435

Gosset, E., Rauw, G., Vreux, J.-M., Nazé, Y., Antokhin, I., \& Sana, H. 2003, in "A Massive Star Odyssey, from Main Sequence to Supernova", Proc. IAU Symp. 212, eds. K.A. van der Hucht, A. Herrero \& C. Esteban, in press

Harries, T.J., Hilditch, R.W., \& Hill, G. 1998, MNRAS, 295, 386

Herrero, A., Kudritzki, R.P., Vilchez, J.M., Kunze, D., Butler, K., \& Haser, S. 1992, A\&A, 261, 209 
Herrero, A., Puls, J., \& Villamariz, M.R. 2000, A\&A, 354, 193

Hill, G.M., Moffat, A.F.J., St-Louis, N., \& Bartzakos, P. 2000, MNRAS, 318, 402

Humphreys, R.M., \& McElroy, D.B. 1984, ApJ, 284, 565

Lamontagne, R., Moffat, A.F.J., Drissen, L., Robert, C., \& Matthews, J.M. 1996, AJ, 112, 2227

Lebedev, M.G., \& Myasnikov A.V. 1988, in "Numerical Methods in Aerodynamics", eds. V.M. Paskonov, G.S. Roslyakov, Moscow State University Press, Moscow, 3

Le Teuff, Y.H. 2002 in "Interacting Winds from Massive Stars", eds. A.F.J. Moffat \& N. St-Louis, ASP Conf. Series 260, 223

Levato, H., Malaroda, S., Morrell, N., Garcia, B., \& Hernández, C. 1991, ApJS, 75,869

Longair, M.S. 1992, "High Energy Astrophysics, Vol. 1: Particles, Photons and their Detection", Cambridge University Press

Luo, D., McCray, R., \& MacLow, M.-M. 1990, ApJ, 362, 267

Maeder, A., \& Meynet, G. 1994, A\&A, 287, 803

Marchenko, S.V., Moffat, A.F.J., Eenens, P.R.J., Cardona, O., Echevarria, J., \& Hervieux, Y. 1997, ApJ, 485, 826

Martins, F., Schaerer, D., \& Hillier, D.J. 2002, A\&A, 382, 999

Massey, P., \& Johnson, J., 1993, AJ, 105, 980

Massey, P., Penny, L.R., \& Vukovich, J., 2002, ApJ, 565, 982

Mayer, P., Harmanec, P., Lorenz, R. et al. 2001 in "The Influence of Binaries on Stellar Population Studies", ed. D. Vanbeveren, Kluwer Academic Publisher, 567

Meynet, G., \& Maeder, A. 2000, A\&A, 361, 101

Morrell, N.I., Barbá, R.H., Niemela, V.S. et al. 2001, MNRAS, 326, 85

Mücke, A., \& Pohl, M. 2001, in "Interacting Winds from Massive Stars", eds. A.F.J. Moffat \& N. St-Louis, ASP Conf. Series 260, 355

Penny, L.R., Gies, D.R., \& Bagnuolo, W.G.Jr. 1996 in "Wolf-Rayet Stars in the Framework of Stellar Evolution", Proc. 33rd Liège Intern. Astroph. Coll., eds. J.-M. Vreux, A. Detal, D. Fraipont-Caro, E. Gosset \& G. Rauw, 289

Pittard, J.M. 2000, PhD thesis, University of Birmingham

Pittard, J.M., \& Stevens, I.R. 1997, MNRAS, 292, 298

Pittard, J.M., Stevens, I.R., Corcoran, M.F., Gayley, K.G., Marchenko, S.V., \& Rauw, G. 2000, MNRAS, 319, 137

Pittard, J.M., Stevens, I.R., Williams, P.M., Pollock, A.M.T., Skinner, S.L., Corcoran, M.F., \& Moffat, A.F.J. 2002, A\&A, 388, 335

Pollock, A.M.T. 1987, ApJ, 320, 283

Prilutskii, O., \& Usov., V. 1976, Sov. Ast.-AJ, 20, 2

Rauw, G. 1997, PhD thesis, Université de Liège 
Rauw, G., Vreux, J.-M., Gosset, E., Hutsemékers, D., Magain, P., \& Rochowicz, K. 1996, A\&A, 306, 771

Rauw, G., Vreux, J.-M., \& Bohannan, B., 1999, ApJ, 517, 416

Rauw, G., Sana, H., Antokhin, I.I. et al. 2001a, MNRAS, 326, 1149

Rauw, G., Nazé, Y., Carrier, F., Burki, G., Gosset, E., \& Vreux, J.-M., 2001b, A\&A, 368, 212

Rauw, G., Vreux, J.-M., Stevens, I.R., Gosset, E., Sana, H., Jamar, C., \& Mason, K.O., 2002a, A\&A, 388, 552

Rauw, G., Crowther, P.A., Eenens, P.R.J., Manfroid, J., \& Vreux, J.-M. 2002b, A\&A, 392, 563

Romero, G.E., Benaglia, P., \& Torres, D.F. 1999, A\&A, 348, 868

Sana, H., Rauw, G., \& Gosset, E. 2001, A\&A, 370, 121

Schaller, G., Schaerer, D., Meynet, G., \& Maeder, A. 1992, A\&AS, 96, 269

Schweickhardt, J., Schmutz, W., Stahl, O., Szeifert, T., \& Wolf, B. 1999, A\&A, 347,127

Skinner, S.L., Güdel, M., Schmutz, W., \& Stevens, I.R. 2001, ApJ, 558, L113

Stevens, I.R., Blondin, J.M., \& Pollock, A.M.T. 1992, ApJ, 386, 265

Stevens, I.R., \& Pollock, A.M.T. 1994, MNRAS, 269, 226

Stevens, I.R., Corcoran, M.F., Willis, A.J., Skinner, S.L., Pollock, A.M.T., Nagase, F., \& Koyama, K. 1996, MNRAS, 283, 589

Stickland, D.J., Lloyd, C., Koch, R.H., \& Pachoulakis, I. 1995, Observatory, 115, 317

Tuthill, P., Monnier, J., \& Danchi, W. 1999, Nat, 398, 487

Vacca, W.D., Garmany, C.D., \& Shull, J.M. 1996, ApJ, 460, 914

Vanbeveren, D., \& de Loore, C. 1994, A\&A, 290, 129

van der Hucht, K.A. 2001, New Astronomy Reviews, 45, 135

van der Hucht, K.A. 2002, in "Multifrequency Behaviour of High Energy Cosmic Sources", eds. F. Giovanelli \& L. Sabau-Graziati, Mem. S. A. It., 73

Walborn, N.R., \& Blades, J.C. 1997, ApJS, 112, 457

Walborn, N.R., Howarth, I.D., Lennon, D.J. et al. 2002, AJ, 123, 2754

Walder, R., \& Folini, D. 2003, in "A Massive Star Odyssey, from Main Sequence to Supernova", Proc. IAU Symp. 212, eds. K.A. van der Hucht, A. Herrero \& C. Esteban, in press

White, R.L., \& Becker, R.H. 1995, ApJ, 451, 352

White, R.L., \& Chen, W. 1992, ApJ, 387, L81

Wiggs, M.S., \& Gies, D.R. 1992, ApJ, 396, 238

Wiggs, M.S., \& Gies, D.R. 1993, ApJ, 407, 252

Williams, P.M. 2002 in "Interacting Winds from Massive Stars", eds. A.F.J. Moffat \& N. St-Louis, ASP Conf. Series 260, 311

Williams, P.M., Dougherty, S.M., Davis, R.J., van der Hucht, K.A., Bode, M.F., \& Setia Gunawan, D.Y.A. 1997, MNRAS, 289, 10

Willis, A.J., Schild, H., \& Stevens, I.R. 1995, A\&A, 298, 549 